\title{
Prevalence of Distal Caries in Mandibular Second Molar Related to Impacted Third Molars in RAKCODS
}

\author{
Aya Khanji*, Sabrin Ali and Carolina Duarte \\ Department of Dentistry, RAK medical and health Sciences University, United Arab \\ Emirates
}

*Corresponding author: Aya Khanji, Department of Dentistry, RAK medical and health Sciences University, United Arab Emirates, Tel: 0506001521; Email: ayakhanji@hotmail.com

\section{Research Article \\ Volume 3 Issue 4}

Received Date: November 23, 2018

Published Date: December 18, 2018

DOI: $10.23880 /$ oajds-16000211

\section{Abstract}

Background: Failure of normal tooth eruption due to various factors is referred to as impaction. The most common impacted tooth is mandibular third molars. It is difficult to treat, and it is indicated to be removed without delay to avoid caries development in the neighboring second molar and loss of both teeth. Extraction of the third molar results in less complications and better dental health, improved gum health in the area adjacent to the second molar.

Aim: The aim of this study was to evaluate the prevalence of distal caries in second molar teeth due to impacted third molar teeth in RAKCODS and their relation to gender and age.

Materials and Methods: A total of 100 Orthopantomograms (OPGs) of patients reporting to RAKCODS over a period of three years were screened and randomly selected according to inclusion and exclusion criteria. The data was collected, and cross checked for any discrepancies and entered into excel spread sheet. Descriptive analysis of the data was done, and results were displayed as graphs.

Results: According to this study, 20\% cases show distal caries on mandibular second molars and $80 \%$ cases show healthy second molars. There was no relation between gender and impaction, but when studied independently, it showed that mesial impaction was more common in males, and horizontal impaction was more common in females. Age wise, there was no relation between age and cavity existence.

Conclusion: A total of $80 \%$ of the patients with impacted third molars had no distal caries on mandibular second molar. It was found that there is no relation between gender and impaction, and that there are equal chances of caries existence regardless of age or gender.

Keywords: Impacted third molar; Distal caries; Radiographic study; Orthopantomograms 


\section{Open Access Journal of Dental Sciences}

\section{Introduction}

Impaction is the failure of normal tooth eruption due to various factors such as an obstacle in the eruption path, abnormal tooth position; lack of space etc. The most common impacted tooth is mandibular third molar or wisdom tooth. It is difficult to treat, and it is indicated to be removed without delay to avoid caries development in the neighboring second molar and loss of both teeth. Forward tilting impacted third molar is partially exposed in the oral cavity causing gingival recession thus exposing neck of the tooth which in return aids in bacterial accumulation that result in distal caries of the neighboring molar. Extraction of the wisdom tooth results in less complications and better dental health, improved gum health in the area adjacent to the second molar.

\section{Review of the Literature}

Third molars are the last teeth to erupt, thus they have inadequate space for eruption [1]. Mandibular third molars erupt at 17 to 21 years age and are most commonly detected in patients in their third decade of life $[2,3]$. Impaction is more frequency in the mandible than maxilla, and ore more often observed unilaterally than bilaterally [1-3]. Impacted mandibular third molars can be categorized based on the Pell and Gregory classification as vertical, mesioangular, distoangular and horizontal [1-4]. Rotation from the mesioangular to the vertical direction is the most common reason for tooth impaction among many others including position and size of adjacent teeth, dense overlying bone, excessive soft tissue or a genetic abnormality including abnormal eruption path, dental arch length, pain, infection, cysts, tumors, jaw fractures, or malposition of the mandibular anterior teeth, resorption of the adjacent teeth or periodontal bone loss [1-3]. Additionally, during clinical examination and radiographs, two types of impactions are found; completely and partially. The complete prevention of normal functional tooth eruption covered by bone and mucosa is called complete impaction while partially exposed tooth to the oral cavity but failure to erupt fully into a normal position is partial impaction [1].

Many impacted third molars remain asymptomatic for years and pose a risk for second molar incident pathology $[3,5]$. Dental caries was the most common pathology associated with impacted mandibular third molars [2]. The survival of second molars depends on third molar status. Second molars adjacent to erupted third molars were more likely to have distal caries than second molars adjacent to absent third molars, and higher in partially impacted than completely impacted $[3,6]$. The most prone to develop caries to adjacent tooth is the partially impacted 3rd molar which doesn't participate in mastication but offers a more favorable environment for bacterial accumulation than fully impacted [1,3]. In some cases, tooth decay in the form of so called "hidden caries" might be present which is characterized by lesion in demineralized dentin that is missed on a visual examination, but is detected radiographically $[1,3]$. The major reason for third molar extraction due to its pathological features is caries and its sequelae [1,2]. In case of partially exposed mesioangular and horizontal mandibular third molars, occlusal surface form plaque accumulative crevices against the distal surfaces of the second molars which develops distal caries in second molars over time. As the gingival margin recedes, CEJ becomes exposed, forming a bacterial retention site leading to formation of root surface caries $[1,3]$. Contact point localization above the amelo-cemental junction, poses less risk than the other positions [3].

In asymptomatic partially impacted third molar in the vertical position with caries development, conservative filling is placed, and, in order to avoid recurrent caries, accessibility to the contact point between the second and third molars which causes the risk of developing distal cervical caries and the patient's good hygiene without dental plaque must be present $[1,3]$. If third molar is left untreated it will result in loss of both teeth $[1,3]$. If the second molar has caries or large restoration, or has been endodontically treated, removal of partially or completely impacted wisdom teeth, must be safely performed without injuring the second molar [1]. In extreme cases, the tooth decay might be developed with great extension on the partially impacted third molars and adjacent second molars that could not be repaired resulting in the extraction of both teeth. However, the most performed surgical procedure, which is the prophylactic removal of asymptomatic third molars that could prevent distal caries formation is neither cost-free nor risk-free [1,3-5].

This study aims to review the incidence of distal caries in second mandibular molar due to impacted third molar tooth. The objectives will be to assess the most common type of third molar impaction associated with cervical caries of second molar tooth, the most common age group with highest incidence of distal caries involving second molars, and the gender variation in the incidence of distal caries involving second molar teeth.

This study aims to review the incidence of distal caries in second mandibular molar due to impacted third molar tooth and to assess associated risk factors. 


\section{Open Access Journal of Dental Sciences}

\section{Materials and Methods}

Radiographic assessment of patients reporting to RAK Collage of Dental Sciences, RAK, UAE has been done. A total of 100 Orthopantomogram's (OPGs) of patients over a period of three years were screened and randomly selected according to the inclusion criteria. Each OPG was analyzed by a minimum of two investigators. OPGs with impacted third molar teeth were identified. Of these OPGs with impacted third molar teeth, prevalence of distal caries in mandibular second molars were assessed and analyzed in relation to age and gender. An excel spread was designed to enter the data. Specific type of impacted third molar causing distal caries in second molar was identified and recorded. Impacted mandibular third molar were classified as per the standard Pell \& Gregory classification as vertical, mesioangular, distoangular, horizontal and others [7-12].

\section{Statistical Analysis}

The data was cross checked for any discrepancies and then subsequently analyzed. Results are displayed as prevalence of distal caries in second molar teeth due to impacted third molars in relation to age, gender and type of impaction. Results displayed as graphs. Correlation coefficient calculated by using SPSS 2.0 version.

\section{Inclusion Criteria:}

- All patients between age group 21 and above

- Patients normal eruption pattern of second molar teeth

- Patient records between "2015 - 2017" are screened.

\section{Exclusion Criteria:}

- Patients who have already extracted third molars

- Patients with congenitally missing third molars

- Patients with implants in second molars

- Patients with associated pathologies like cysts, tumors

- Patients with developmental disorders like microdontia, presence of 4th molars, impacted second molars, supernumerary teeth and odontomas.

\section{Results}

There was no relation between gender and cavity existence. However, when genders were compared separately, it was found that mesial impaction was more common in males than females. In addition, it was found that horizontal impaction was more common is females than males (Figures 1-6 \& Table 1).

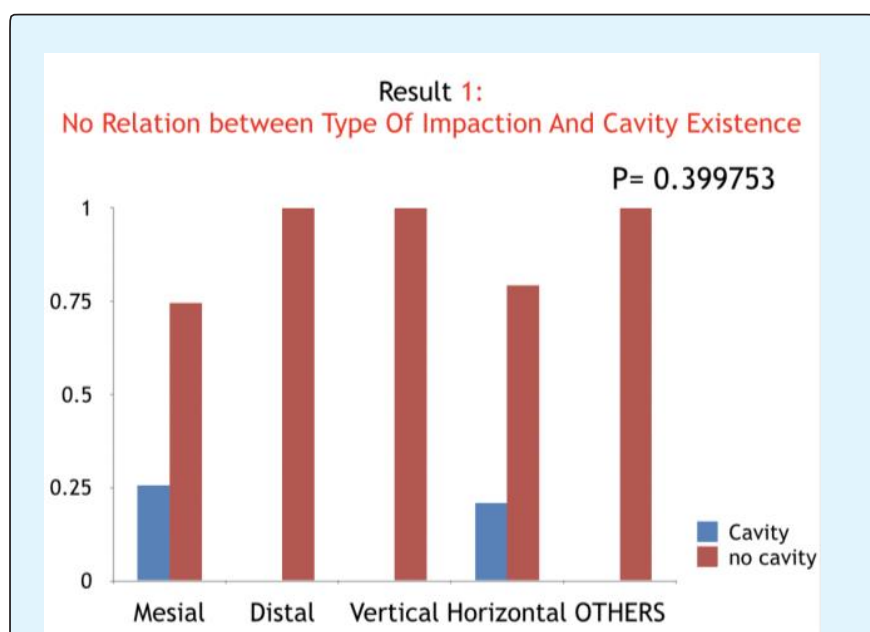

Figure 1: No relation between type of impaction and cavity existence.

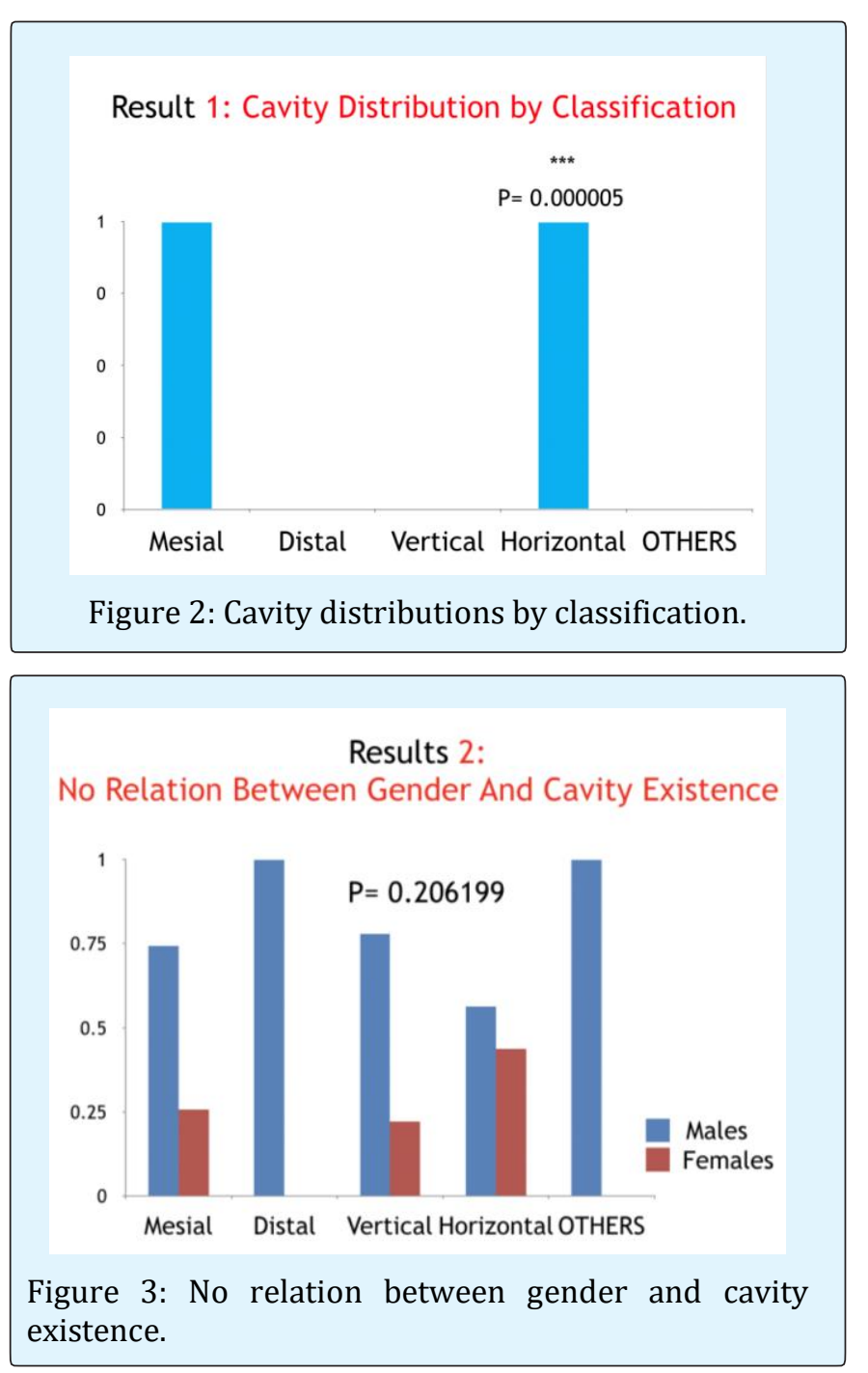



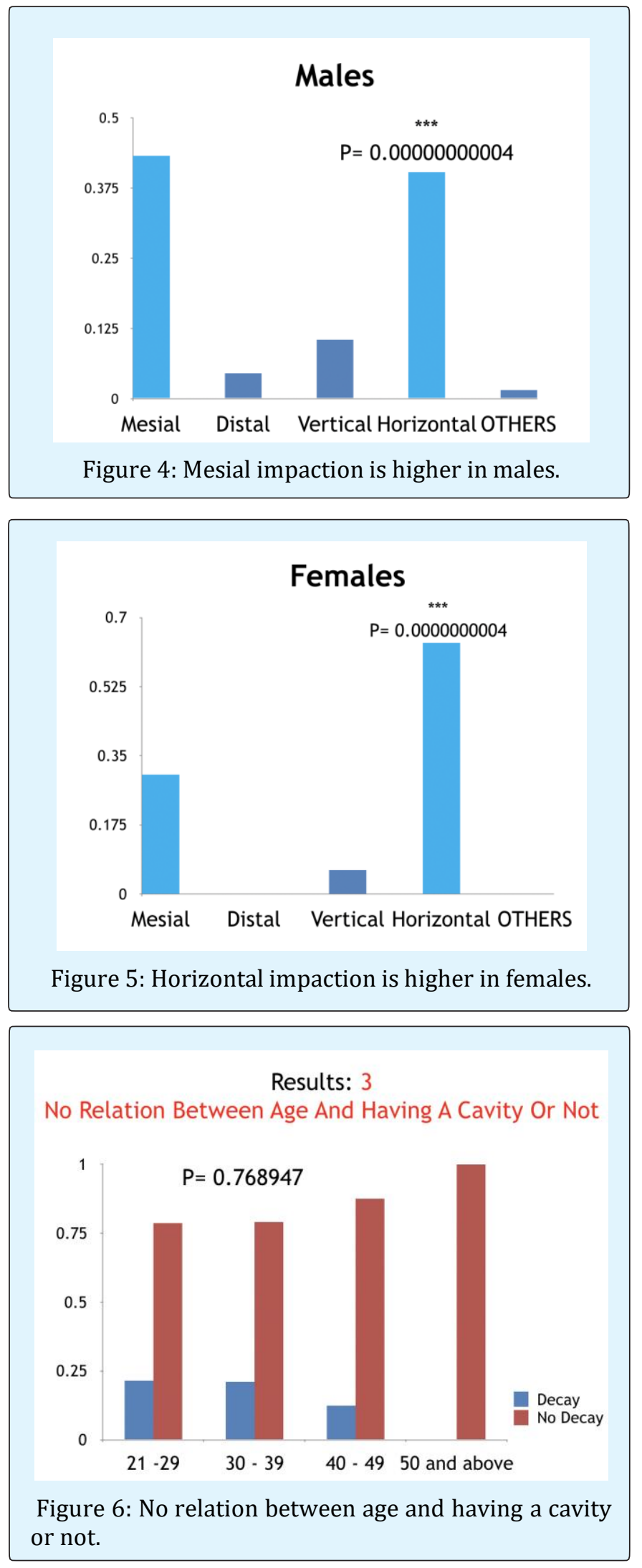

\begin{tabular}{|c|c|c|c|}
\hline & AGE & GENDER & Decayed \\
\hline GENDER & $-0.18 \mathrm{~ns}$ & & \\
\hline Decayed & $-0.14 \mathrm{~ns}$ & $-0.09 \mathrm{~ns}$ & \\
\hline Type of Impaction & $0.03 \mathrm{~ns}$ & $-0.02 \mathrm{~ns}$ & $-0.19^{*}$ \\
\hline
\end{tabular}

Table 1: Correlation coefficient, *significant at 0.05 .

Correlation analysis conducted between different variables of the study (age, gender, decay existence and impaction). Significance is only shown between impaction and existence of decay with a value of 0.19 at 0.05 level of significance which means that decay existence is related to type of impaction of the mandibular third molar, and this is regardless of age and gender.

\section{Discussion}

Impaction of the third molar is a very common phenomenon. Distal caries of mandibular second molar is a frequently noted complication of impacted third molars. The total number of the patients admitted to RAKCODS in years between 2015 to 2017 years was (7832) and among them, a number of (100) OPGs were taken from them. In this study, it was found that there was no relation between impaction and cavity existence (80\%). However, if it was studied independently, it would show that there was a particular prevalence of mesial and horizontal impaction because they were the most common types in the group (20\%) [13-19].

A total of 100 patients had impacted third molars $(1.276 \%)$. A total of $80 \%$ patients with impacted third molars had no distal caries in second molar but, a total of $20 \%$ patients had distal caries in second molar. In those who had distal caries, mesioangular and horizontal impactions were the most prominent types. There was no relation between gender and impaction type. However, when studied independently, males who had distal caries were more likely to have mesial impaction while females who had distal caries had horizontal impaction. Age wise, as per the study, there was no relation between cavity existence and age. Therefore, there was more prevalence of healthy teeth regardless of age.

\section{Conclusion}

A total of $80 \%$ patients with impacted third molars had no distal caries in second molar and a total of $20 \%$ patients had distal caries in second molar. There was no relation between gender and impaction type. In addition, there was no relation between age and having a cavity or not. 


\section{Open Access Journal of Dental Sciences}

Acknowledgement: I am grateful to my supervisor Dr. Sabrin Ali and my coordinator Dr. Carolina Duarte for helping and guiding me to finish this research in different matters. I am thankful to everyone who supported me in this research and gave me their guidance to complete my work effectively and moreover on time.

\section{References}

1. Jung YH, Cho BH (2013) Prevalence of missing and impacted third molars in adults aged 25 years and above. Imaging Sci Dent 43(4): 219-225.

2. Asif SA, Syed N, Shah AA, Akhtar MU (2014) Dental Caries and Pericoronitis associated with Impacted Mandibular Third Molars-A Clinical and Radiographic Study. Pakistan Oral \& Dental Journal 34(2): 268-273.

3. Brkić A (2012) Impacted Teeth and Their Influence on the Caries Lesion Development.

4. Hashemipour MA, Tahmasbi Arashlow M, Fahimi Hanzaei F (2012) Incidence of impacted mandibular and maxillary third molars: a radiographic study in a Southeast Iran population. Med Oral Patol Oral Cir Bucal 18(1): 140-145.

5. Juodzbalys G, Daugela P (2013) Mandibular third molar impaction: review of literature and a proposal of a classification. J Oral Maxillofac Res 4(2): e1.

6. Byahatti S, Ingafou MS (2012) Prevalence of eruption status of third molars in Libyan students. Dent Res J 9(2): 152-157.

7. Khawaja NA, Khalil H, Parveen K, Al Mutiri A, Al Mutiri, et al. (2015). A Retrospective Radiographic Survey of Pathology Associated with Impacted Third Molars among Patients Seen in Oral \& Maxillofacial Surgery Clinic of College of Dentistry, Riyadh. J Int Oral Health 7(4): 13-17.

8. Marciani RD (2012) Is there pathology associated with asymptomatic third molars? J Oral Maxillofac Sur 70(9): 15-19.

9. Msagati F, Simon EN, Owibingire S (2013) Pattern of occurrence and treatment of impacted teeth at the Muhimbili National Hospital, Dar es Salaam, Tanzania. BMC Oral Health 13: 37.
10. Nunn ME, Fish MD, Garcia RI, Kaye EK, Figueroa R, et al. (2013) Retained asymptomatic third molars and risk for second molar pathology. J Dent Res 92(12): 1095-1099.

11. Patil S, Halgatti V, Khandelwal S, Santosh BS, Maheshwari S (2014) Prevalence of cysts and tumors around the retained and unerupted third molars in the Indian population. J Oral Biol Craniofac Res 4(2): 82-87.

12. Prajapati VK, Mitra R, Vinayak KM (2017) Pattern of mandibular third molar impaction and its association to caries in mandibular second molar: A clinical variant. Dent Res J 14(2): 137-142.

13. Santosh P (2015) Impacted Mandibular Third Molars: Review of Literature and a Proposal of a Combined Clinical and Radiological Classification. Ann Med Health Sci Res 5(4): 229-234.

14. Sheikh MA, Riaz M, Shafiq S (2012) Incidence of distal caries in mandibular second molars due to impacted third molars-A clinical and radiographic study. Pakistan Oral \& Dental Journal 32(3): 364-370.

15. Srivastava N, Shetty A, Goswami RD, Apparaju V, Bagga V, et al. (2017) Incidence of distal caries in mandibular second molars due to impacted third molars: Nonintervention strategy of asymptomatic third molars causes harm? A retrospective study. Int J Appl Basic Med Res 7(1): 15-19.

16. Syed KB, Alshahrani FS, Alabsi WS, Alqahtani ZA, Hameed MS, et al. (2017) Prevalence of Distal Caries among Mandibular Second Molar Due to Impacted Third Molar Teeth in Saudi Population. J Clin Diagn Res 11(3): 28-30.

17. Tabrizi R, Arabion H, Gholami M (2013) How will mandibular third molar surgery affect mandibular second molar periodontal parameters?. Dent Res J 10(4): 523-526.

18. Hassan AH (2010) Pattern of third molar impaction in a Saudi population. Clin Cosmet Investig Dent 2: 109113.

19. Vigneswaran AT, Shilpa S (2015) The incidence of cysts and tumors associated with impacted third molars. J Pharm Bioallied Sci 7(1): 251-254.

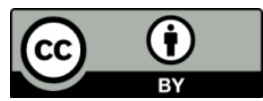

